\title{
Circuit
}

Musiques contemporaines

\section{Le quatuor, le compositeur et le musicologue}

\section{Fable}

\section{Jean Boivin}

Volume 11, numéro 2, 2000

Le quatuor à cordes selon Schafer

URI : https://id.erudit.org/iderudit/004651ar

DOI : https://doi.org/10.7202/004651ar

Aller au sommaire du numéro

Éditeur(s)

Les Presses de l'Université de Montréal

ISSN

1183-1693 (imprimé)

1488-9692 (numérique)

Découvrir la revue

Citer cet article

Boivin, J. (2000). Le quatuor, le compositeur et le musicologue : fable. Circuit, 11(2), 69-72. https://doi.org/10.7202/004651ar d'utilisation que vous pouvez consulter en ligne.

https://apropos.erudit.org/fr/usagers/politique-dutilisation/ 


\title{
Le quatuor, le compositeur et le musicologue. Fable
}

\author{
Jean Boivin
}

Pour qui écriton aujourd'hui des quatuors à cordes? À cette question pertinente, posée par Jean Portugais, je répondrai, sans modestie mais avec une pointe d'humour: mais pour le musicologue, bien sûr! Le quatuor à cordes, genre musical auréolé de gloire, qui est à la musique de chambre ce que le sonnet est à la poésie, a depuis longtemps la faveur de ceux qui ont pour mission d'écrire sur la musique, précisément pour les raisons données par Pierre Boulez à la fin du texte soumis aux participants de cette table ronde. Il est en effet généralement admis que le quatuor à cordes, dont les exigences sont grandes sur le plan technique, incite les compositeurs à une démarche plus réfléchie, plus rigoureuse, et à une inspiration particulièrement élevée. D'où l'intérêt du chercheur ou du commentateur pour ce qui constitue la quintessence d'un style, d'une production musicale. À cela s'ajoute le fait que le musicologue apprécie généralement les corpus fermés, ou à tout le moins suffisamment développés pour permettre les comparaisons, d'une part entre les œuvres qu'il contient (l'ensemble des quatuors de Beethoven, par exemple) et, d'autre part, entre celles-ci et les autres œuvres du répertoire (les derniers quatuors de Beethoven en regard de l'ensemble des œuvres de la période classique destinées au quatuor, pour poursuivre l'analogie).

Plusieurs compositeurs contemporains ont eu la bonté d'écrire plusieurs quatuors à cordes (ou des œuvres destinées à cette formation mais portant un autre nom ' l, formant des ensembles que l'on peut étudier, au même titre que les séries illustres de Haydn, Beethoven, Schubert, Bartók ou Chostakovitch. Parmi ces compositeurs généreux figurent Elliott Carter, Giacinto Scelsi, György Kurtág, Milton Babbitt, Michael Tippett, Wolfgang Rihm et Brian Ferneyhough. Sans oublier bien sûr R. Murray Schafer, dont l'intérêt marqué pour le genre ne paraît pas épuisé. Par contre, d'autres compositeurs, à l'exemple de Debussy et de Ravel, ne s'y sont frottés qu'une seule fois, ou deux tout au plus. C'est le cas, pour autant que je sache, de Boulez, de Cage, de Lutoslawski, de Berio, de Nono, de Crumb ${ }^{2}$. Les deux cas de figure sont à mon avis également intéressants et porteurs de messages.
1. Je pense notamment aux Bagatelles de Webern, aux Microludes de Kurtág et aux Sonates de Ferneyhough.

2. Il est intéressant de noter que pour plusieurs d'entre eux, encore une fois à l'exemple de Debussy et de Ravel, ces œuvres ont vu le jour au début de leur carrière de compositeur. Le premier des trois quatuors de Ligeti, composé en 1953 1954, est une œuvre de jeunesse, de la période "hongroise». 
Ceux à qui l'on doit des «recueils» de quatuors s'insèrent tout naturellement dans la lignée des compositeurs prolifiques déjà nommés, dont les œuvres sont devenues des classiques du genre. À tout le moins sommes-nous en droit de supposer qu'ils ont ainsi cherché, plus ou moins délibérément, à joindre leurs rangs, à créer un corpus véritable qui trouve place dans I'histoire du genre ${ }^{3}$. Car la composition d'une série d'œuvres pour une même formation invite en soi à la comparaison, à l'analyse, au classement. À l'ordonnance des œuvres, de la jeunesse à la maturité. À l'identification des étapes successives de la maîtrise du médium, de l'apprentissage du métier au dépassement des considérations techniques et à l'atteinte d'une expression pleinement maîtrisée. Aux autres - moins prolixes - incombent le rôle plus difficile de se démarquer d'un seul coup. Or, on sait à quel point l'originalité demeure une qualité prisée dans le monde artistique contemporain ${ }^{4}$. L'œuvre isolée, le quatuor, est alors observée en considérant à l'arrière-plan le reste de la production du compositeur, de même que l'ensemble du répertoire pour cette formation ${ }^{5}$. Dans un cas comme dans l'autre, le genre hautement " historicisé » du quatuor permet au compositeur, comme le suggère le musicologue américain James McCalla, d'entrer en conversation avec ses collègues, proches ou lointains. S'établit alors un curieux dialogue sur la musique et en musique, dans le temps et en temps (entendre: en temps réel). Ce bras de fer avec le quatuor, croit McCalla, permet au compositeur de se joindre à tous ceux qui se sont adressés à lui par le passé, d'entendre ce qu'ils ont à dire avant d'ajouter sa propre contribution (McCalla, 1996, p. 184). Une idée qui ne déplaira pas, j'imagine, à John Rea, qui participe à cette discussion et qui entretient lui-même avec I'histoire de la musique des apartés stimulants pour sa propre création let pour nous...). Ce dialogue se révèle pour ainsi dire un cas d'affaire publique, puisqu'un quatuor "réussi » vaudra au compositeur le respect de ses pairs, du public et de la critique ${ }^{6}$.

Le poids de la tradition est lourd, sans doute, mais peut-être ce fardeau est-il justement compensé par l'envie ressentie par plusieurs de se mesurer à celle-ci, de se positionner par rapport au répertoire existant, à l'occasion en s'y référant directement (tout, en musique, n'est-il pas une question de mémoire?). Le défi à relever pourra se révéler une fin en soi. Pensons à Ravel ou à Stravinsky, qui se sentaient tous deux stimulés par les contraintes. Il est pratiquement impossible, en fait, d'ignorer l'histoire. Autant "faire avec», comme on dit en langage courant. Le compositeur qui entreprend l'écriture d'un quatuor à cordes consultera, plus souvent qu'autrement, les réalisations antérieures et reviendra, comme par un aimant attiré, vers Beethoven, Bartók ou Carter, les grands maîtres du genre (noms mythiques auxquels d'aucuns voudront sans doute déjà ajouter celvi de Schafer puisque tout compositeur canadien qui tentera d'entreprendre une série importante d'œuvres pour cette formation devra dorénavant accepter de lui être comparé).

Un corpus de quatuors à cordes, une série d'œuvres réparties plus ou moins régulièrement dans une carrière, offre par ailleurs une fenêtre privilégiée pour
3. On pourrait ainsi "expliquer» les dix-huit quatuors de Darius Milhaud. Villa-Lobos en a pour sa part composé seize.

4. Remarquons au passage que cette notion n'importait guère au temps de Telemann ou du jeune Mozart, lesquels ne se sentaient nullement gênés par l'acte d'emprunt ou d'hommage.

5. Ainsi en est-il de Boulez qui, après s'être commis dans la sonate, a choisi d'écrire un Livre pour quatuor à cordes, une œuvre pivot dans sa démarche.

6. L'exemple du quatuor de Serge Provost, joué par le Quatuor Arditti, est éloquent. 
observer l'évolution d'un compositeur, son degré de sensibilité aux influences, la manière dont il absorbe les acquis, les réinterprète, fait siennes les trouvailles qui lui «parlent» directement, et rejettent d'autres avenues, sans nécessairement les condamner. Sur un plan plus pratique (et avec un sourire au coin des lèvres), je ne peux m'empêcher de remarquer qu'il est facile, que l'on soit compositeur ou appelé à commenter le travail de création de celvi-ci, de se déplacer avec la partition d'un quatuor en poche. Ainsi en fut-il de Messiaen qui allégea les longues heures d'emprisonnement dans un stalag grâce à l'étude minutieuse des quatuors de Beethoven, ou de Clermont Pépin qui vécut un temps dans le voisinage direct de la Suite lyrique de Berg. Eł qui sait combien d'études musicologiques éclairées et consacrées aux quatuors de Schönberg ont été conçues ou même rédigées dans un train, un avion ou un autobus, voire sur la plage? Certains créateurs semblent toutefois avoir voulu parer le coup, et se sont épanchés en de fort volumineuses et lourdes partitions. Je pense aux véritables pavés de Wolfgang Rihm, dont on ne pourrait rendre compte que par une quantité tout aussi appréciable de pages de texte...

À ce propos, on a souvent décrit les compositions sérielles plutôt abstraites datées des années cinquante comme une musique à lire plutôt qu'à entendre. (On parlait aussi alors de "compositeurs de tableaux noirs»). Le quatuor à cordes contemporain combine, à mon avis, de manière particulièrement heureuse ces deux façons d'appréhender une production, la vue s'associant à l'ouie, l'analyse à la perception. R. Murray Schafer, dont les partitions peuvent être considérées comme de véritables œuvres d'art, concédera sans doute qu'il est plus facile d'organiser un événement centré sur sa musique de chambre que sur ses œuvres scéniques ou à portée environnementale, élaborées à partir d'un paysage sonore donné et qui ne peuvent être transmises que très partiellement, au prix d'énormes compromis, par une partition ou un enregistrement sur disque compact, ou même sur une bande vidéo. Ces œuvres ne risquenteelles pas, justement pour cette raison, d'être sous-évaluées a posteriori dans l'ensemble de sa production?

Je terminerai sur une réflexion, ou plutôt une interrogation, en apparence sans lien avec ce qui précède. Tout comme la «réduction » d'une vaste symphonie pour piano, piano à quatre mains ou deux pianos, le quatuor à cordes fait souvent office de cliché en noir et blanc du style d'un compositeur. Car ici point de facilité, de maquillage abusif par le timbre, d'opération de charme, qui ne transparaisse immédiatement. Quoi qu'on puisse penser de l'élévation du quatuor sur un piédestal, de son statut de Walhalla de la création "sérieuse", pour reprendre l'expression de Jean Portugais (ou de Parnasse musical, selon McCalla), il demeure que l'écriture pour le quatuor implique un certain ascétisme de la pensée, et souvent une attention particulière à la dimension polyphonique, en vertu de la transparence que permet l'écriture à quatre voix ${ }^{7}$.

Et pourquoi, quatre voix, au fait? Par pur respect d'une tradition dont l'origine remonte à la jeunesse de Haydn, et même plus loin si l'on songe à ce qui perdure
7. II existe bien sûr de nombreuses exceptions à ce que l'on peut considérer comme une présomption polyphonique, certains quatuors étant volontairement axés sur l'homophonie. 
dans l'écriture pour quatuor de la sonate en trio ou du choral harmonisé? Le langage tonal était particulièrement bien servi par l'écriture à quatre voix, ainsi que l'a souligné avec justesse le musicologue américain Charles Rosen : trois voix pour faire entendre les notes de la triade sur laquelle repose l'édifice de la tonalité, et I'autre pour faire dissonance avec celle-ci, la tension créée exigeant un apaisement à court ou moyen terme (Rosen, 1978, p. 174). Déjà, on peut se demander pourquoi Schönberg a respecté cette convention, alors même qu'il abandonnait toute idée de résoudre les dissonances, de préserver le sentiment de polarité tonale. Si la révérence profonde de Schönberg pour la grande tradition germanique, révérence exprimée autant dans sa musique que dans ses écrits, nous fournit un élément de réponse, pourquoi Boulez, Cage, Kurtág, Schafer et Provost en ont-ils fait autant? Mozart savait que le trio à cordes peut se révéler encore plus exigeant, et Schubert a fait les merveilles que l'on sait avec un quintette comprenant deux violoncelles. Alors pourquoi, encore et toujours, le quatuor ${ }^{8}$ ?

\section{Bibliographie}

McCALLA, J. (1996), Twentieth-Century Chamber Music, New York, Schirmer, coll. "Studies in Musical Genres and Repertoire», 274 p.

ROSEN, C. (1978), Le style classique, Haydn, Mozart, Beethoven, trad. de l'anglais par M. Vignal, Paris, Gallimard, coll. «Bibliothèque des idées», 592 p.

8. On pourra bien sûr suggérer un élément de réponse qui ne me satisfait toutefois pas pleinement. On écrit des quatuors pour être joué par les ensembles voués au large répertoire pour cette formation. Rares en effet sont les compositeurs qui se lancent aujourd'hui avec enthousiasme dans la composition d'une nouvelle œuvre de dimensions importantes sans l'assurance qu'elle sera exécutée, ou du moins sans de bonnes raisons d'espérer qu'elle le sera. Or, on sait que les contingences économiques et les agendas chargés des musiciens rendent la préparation d'œuvres conçues pour de larges ensembles délicate à planifier et, qui plus est, fort onéreuse. Les musiciens qui se consacrent au quatuor à cordes ont, à l'opposé, la réputation d'être stables, disciplinés et moins avares de leur temps. Les heures de travail qu'ils (ou elles) consacreront à une partition nouvelle len commun ou sur le plan individuel) seront rarement rétribuées en totalité. Entre ici une bonne part de dévouement. Voilà un point en faveur du quatuor à cordes. Un compositeur ayant dans son entourage un ensemble de qualité disposé à interpréter des œuvres récentes se verra encouragé à écrire une nouvelle œuvre pour cette formation, qu'il se sente "appelé » par elle ou non. 